\title{
THE PROPERTY OF UNIVERSALITY FOR PURE PERMANENT MAGNET WIGGLERS
}

\author{
M. N. Smolyakov", Physical Department, Moscow State University, Moscow, Russia
}

\section{Abstract}

This paper deals with electromagnetic radiation generated by relativistic particles in arbitrary planar magnetic field (in a wiggler or undulator for example). Magnetic system producing this field is assumed to be planar and to consist of permanent magnets. It is shown that there is a special rotations for vectors of magnetization in such magnetic system: while magnetic field is varying but the spectrum of spontaneous radiation generated by relativistic particles remains the same. This property of radiation can be used in design of the new undulators and wigglers.

\section{INTRODUCTION}

Nowadays, permanent magnets are widely used in wiggler and undulator designs. Only permanent magnets are used in pure permanent magnet (PPM) wigglers [1]. Sometimes unusual undulator designs are used, especially for the microundulators [2-5]. To enhance the FEL gain, the non-standard scheme was chosen also for the PPM wiggler [6,7], i.e. with the magnetization vectors parallel and anti-parallel to the wiggler axis. That is why it is very important to investigate the general properties of the planar PPM undulators/wigglers and the electromagnetic radiation generated by relativistic electron beam in such systems. In particular the Rotation Theorem is well known [8]: if at each point of a PPM system all easy axis are rotated simultaneously by the angle $\theta$, while the geometry remains the same, then all magnetic fields rotate by the opposite angle $-\theta$ without the change in amplitude.

This paper also deals with another general property of any planar PPM wiggler/undulator. It is shown that if all easy axis of the upper part of PPM undulator are rotated by the angle $\theta$, and at the same time all easy axis of the bottom part of PPM undulator are rotated by the opposite angle $-\theta$ the shape of magnetic field clearly varies, but the module of it's Fourier transform remains constant.

\section{FOURIER TRANSFORM INVARIANCE PROPERTY FOR MAGNETIC FIELD}

Let us consider a PPM system that is infinitely wide and homogeneous along the $X$-axis (horizontal axis) with

\footnotetext{
"Email: nick@ smolyakov.msk.ru
}

magnetization $\vec{M}(y, z)$, see Fig.1. Magnetic field produced by such a system is given by [9]:

$$
\vec{H}(\vec{r})=\int_{V} d^{3} \vec{r}^{\prime} \frac{3 \vec{R}\left(\vec{M}\left(\vec{r}^{\prime}\right) \cdot \vec{R}\right)-\vec{M}\left(\vec{r}^{\prime}\right) R^{2}}{R^{5}},
$$

where $\vec{R}=\vec{r}-\vec{r}^{\prime}, R=|\vec{R}|$.

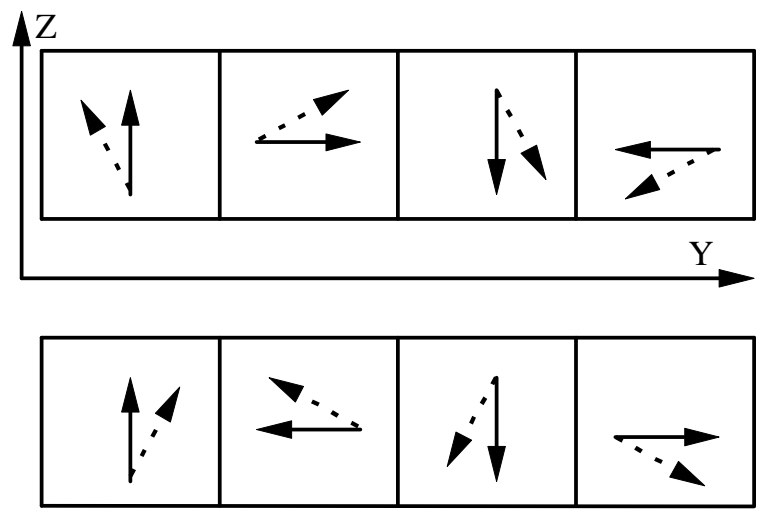

Figure 1: Rotation of easy axis in planar PPM wiggler: solid arrows show the initial magnetization, dashed arrows show the magnetization after rotation. Standard period of PPM wiggler is used as an example.

Let us consider the vertical magnetic field Fourier transform in the wiggler median plane $(z=0)$ :

$\tilde{H}_{z}(p)=\int_{-\infty}^{\infty} e^{i p y} H_{z}(y, z=0) d y$.

Substituting (1) into (2) and making some analytical calculations yields the relationship [10]:

$\widetilde{H}_{z}(p)=2 \pi \int A \exp \left(-i p y^{\prime}-\left|p z^{\prime}\right|\right) d y^{\prime} d z^{\prime}$,

$A=|p| M_{z}\left(y^{\prime}, z^{\prime}\right)-i p M_{y}\left(y^{\prime}, z^{\prime}\right) \operatorname{sign}\left(z^{\prime}\right)$.

Let us rotate the easy axis at each point of upper part of the PPM system $\left(z^{\prime}>0\right)$ by the angle $\theta$ :

$$
\begin{aligned}
& M_{y}^{\prime}(y, z)=M_{y}(y, z) \cos (\theta)-M_{z}(y, z) \sin (\theta), \\
& M_{z}^{\prime}(y, z)=M_{z}(y, z) \cos (\theta)+M_{y}(y, z) \sin (\theta) .
\end{aligned}
$$

As a result we will find that at $\left(z^{\prime}>0\right)$ and $p>0$ the function $A$ will obtain the additional phase factor:

$A^{\prime}=A \exp (-i \theta)$

The rotation of the easy axis at each point of down part of the PPM system $\left(z^{\prime}<0\right)$ by the angle $-\theta$ is given by: 


$$
\begin{aligned}
& M_{y}^{\prime}(y, z)=M_{y}(y, z) \cos (\theta)+M_{z}(y, z) \sin (\theta), \\
& M_{z}^{\prime}(y, z)=M_{z}(y, z) \cos (\theta)-M_{y}(y, z) \sin (\theta) .
\end{aligned}
$$

It is easy to see that the integral (3) over the bottom part of PPM system $\left(z^{\prime}<0\right)$ will get the same phase factor as (5). As a result we have at $p>0$ :

$$
\tilde{H}_{z}^{\prime}(p)=\tilde{H}_{z}(p) \exp (-i \theta) \text {. }
$$

Using the similar calculations, we have at $p<0$ :

$$
\tilde{H}_{z}^{\prime}(p)=\widetilde{H}_{z}(p) \exp (i \theta) \text {. }
$$

Magnetic field is a real function. It is evident from (6) and (7), that the complex conjugation of its Fourier transform is equivalent to replacing $p$ by $-p$.

Eqs. (6) and (7) shows that if all the easy axis at each point of the upper part of PPM system are rotated by the angle $\theta$ and at the same time all the easy axis at each point of the bottom part of PPM system are rotated by the opposite angle $-\theta$, the module of Fourier transform of the magnetic field does not change (Fig. 1). But the phase factors in Fourier transform at $p>0$ and $p<0$ are different. It means that the shape of the magnetic field will be changed.

A brief analysis was made in [6] for the different magnetic fields produced by two different PPM undulators. Actually the second undulator in [6] could be derived from the first one by rotation of easy axis by the angle $-\pi / 2$ at upper part of this undulator and by the angle $\pi / 2$ at its bottom part. Results obtained in [6] are in excellent agreement with results obtained above in this chapter.

\section{INVARIANCE OF ELECTROMAGNETIC RADIATION}

Let us consider the spontaneous electromagnetic radiation, generated by relativistic particle in planar magnetic fields. At first, let us consider the case of relatively weak magnetic field. It means that the angle by which the particle is deflected by the magnetic field is small in comparison with the quantity $1 / \gamma$, where $\gamma$ is a reduced energy of relativistic particle. As this takes place, electromagnetic radiation is dipole-type. It means for undulators that the undulator deflection parameter is much smaller than unit. Electromagnetic radiation from the microundulators (undulators with period less than several millimeters) is almost dipole-type. Spectral characteristics of dipole radiation are determined by the module squared of the Fourier transform of the magnetic field vertical component [11]. Let us transform the magnetic system as it was described above in section 2 . Let us rotate the easy axis at each point of the upper part of PPM system by the angle $\theta$ and at each point of the bottom part of PPM system by the opposite angle $-\theta$ (Fig. 1). Using results obtained in section 2 and the property of dipole radiation noted above, we can conclude, that spectral characteristics of electromagnetic radiation of relativistic particle remain invariant under these transformations of PPM magnetic system.

Let us consider the planar PPM undulator with period $l$ and infinite length. Integral of the magnetic field over the undulator period is equal to zero. Radiation wavelength $\lambda$, generated by the harmonic with number $n$ at the angle $\theta$ to the undulator axis, is equal to:

$\lambda=\frac{l}{2 n \gamma^{2}}\left(1+\gamma^{2} \theta^{2}+0.5 K^{2}\right)$.

Deflection parameter $K$ for the nonsinusoidal magnetic field is equal to:

$K^{2}=\frac{2}{l} \gamma^{2} \int_{0}^{l} \beta_{x}^{2}(y) d y$,

where $\beta_{x}(y)$ is a horizontal reduced speed of the particle. It is clear from the equations of motion that:

$\beta_{x}(y)=\frac{e}{m c^{2} \gamma} \int_{0}^{y} H_{z}\left(y^{\prime}\right) d y^{\prime}+\beta_{x}(0)$.

The particle initial conditions were chosen in such a way that trajectory of the particle is a periodic function. The Fourier coefficients for the $\beta_{x}(y)$ function are:

$\beta_{x k}=\frac{1}{l} \int_{0}^{l} \exp \left(i \frac{2 \pi}{l} k y\right) \beta_{x}(y) d y$.

Since the transverse drift of the particle along one undulator period is equal to zero, the Fourier coefficient at $k=0$ equal to zero, i.e. $\beta_{x 0}=0$. Using Parseval theorem, we obtain:

$\frac{1}{l} \int_{0}^{l} \beta_{x}^{2}(y) d y=\sum_{k=-\infty}^{\infty}\left|\beta_{x k}\right|^{2}$.

It is easy to show from (10) and (11) that at $k \neq 0$ Fourier coefficients for the reduced speed and Fourier coefficients for the undulator magnetic field are proportional to each other:

$\beta_{x k}=\frac{i}{2 \pi} \frac{e l}{k m c^{2} \gamma} H_{z k}$,

where $e$ is a particle charge, $m$ is its mass, $c$ is the speed of light,

$H_{z k}=\frac{1}{l} \int_{0}^{l} \exp \left(i \frac{2 \pi}{l} k y\right) H_{z}(y) d y$.

It is easy to obtain the formulae for the undulator magnetic field Fourier coefficients (14), which are similar to Eqs. (3), (4). Using (9) - (14), we can prove the following statement.

We transform now the planar PPM undulator by the method described in section 2. Let us rotate the easy axis at each point of the upper part of PPM undulator by the 
angle $\theta$ and at each point of the bottom part of PPM undulator by the opposite angle $-\theta$ (Fig. 1). As this takes place the Fourier coefficients (14) for undulator magnetic field and the Fourier coefficients (11) for the reduced speed will get the phase factor. It is evident from (9) and (12) that the undulator deflection parameter is invariant under this magnetic system transformation. It means that the position of undulator radiation harmonic does not shift also. But at the same time the intensity of undulator radiation at some harmonic might change, because of undulator magnetic field shape changes. This problem calls for further investigations.

\section{REFERENCES}

[1] K. Halbach, Nucl. Instr. and Meth. 187 (1981) 109.

[2] G. Ramian, L. Elias and I. Kimel, Nucl. Instr. and Meth. A250 (1986) 125

[3] R. Tatchin and P. Csonka, J. Phys. D: Appl. Phys. 20 (1987) 394.

[4] R. Tatchin, P. Csonka and A. Toor, Rev. Sci. Instrum. 60, No.7 (1989) 1796.

[5] I. Kimel and L. Elias, Nucl. Instr. and Meth. A296 (1990) 611.

[6] P.G. O'Shea, S.C. Bender, D.A. Byrd, J.W. Early, D.W. Feldman, C.M. Fortgang, J.C. Goldstein, B.E. Newnam, R.L. Sheffield, R.W. Warren, T.J. Zaugg, Nucl. Instr. and Meth. A341 (1994) 7.

[7] R.W. Warren and C.M. Fortgang, Nucl. Instr. and Meth. A341 (1994) 444.

[8] K. Halbach, Nucl. Instr. and Meth. 169 (1980) 1.

[9] L.D. Landau and E.M. Lifshitz, The Classical Theory of Fields (Pergamon, Oxford, 1971)

[10] N.V. Smolyakov, Nucl. Instr. and Meth. A308 (1991) 80.

[11] R. Coisson, Phys. Rev. A20, No. 2 (1979) 524. 